\title{
Long-term experience with coronary sinus side branch stenting to stabilize left ventricular electrode position
}

\author{
László Gellér, MD, PhD, Szabolcs Szilágyi, MD, PhD, Endre Zima, MD, PhD, Levente Molnár, MD, \\ Gábor Széplaki, MD, PhD, Eszter M. Végh, MD, István Osztheimer, MD, \\ Béla Merkely, MD, PhD, DSc, FHRS
}

From the Heart Center, Semmelweis University, Budapest, Hungary.

\begin{abstract}
BACKGROUND Despite technical advancements, implantation of coronary sinus (CS) leads may be challenging, and dislocation remains a relevant clinical problem.

OBJECTIVE The aim of this study was to investigate the effectiveness, safety, and long-term outcome of stent implantation to anchor the lead to the wall of the CS side branch.
\end{abstract}

METHODS Stenting of a CS side branch was performed in 312 patients. The procedure was performed because of postoperative lead dislocation in 16 patients and because of an intraoperative unstable lead position or phrenic nerve stimulation in 296 cases. A bare metal coronary stent was introduced over a second guide wire in the same CS sheath. The stent was deposited 5-35 mm proximal to the most proximal electrode. Mechanical damage of the CS side branch or pericardial effusion was not observed owing to stenting.

RESULTS During follow-up (median 28.4, interquartile range 1537, maximum 70 months), a clinically important increase in the left ventricular pacing threshold was found in four cases and

\section{Introduction}

Cardiac resynchronization therapy (CRT) plays an important role in the treatment of severe heart failure in patients with wide QRS complex and mechanical dyssynchrony. Despite continuous technical developments in the last few years, implantation of a CRT system may be challenging. The success rate of coronary sinus (CS) lead positioning is $88 \%-96 \%$ in previous clinical studies, ${ }^{1-4}$ but even in the recently published MADIT CRT trial, $7.5 \%$ of the CS lead implantations were unsuccessful. ${ }^{5}$ During follow-up, 5\%$10 \%$ of patients require reoperation because of CS lead dysfunction. ${ }^{2,3,5-7}$

Stent implantation, which anchors the CS electrode to the wall of the CS side branch, may increase the stability of the

This study was supported by the National Development Agency of Hungary (TÁMOP-4.2.2-08/1/KMR-2008-0004) and an NKTH-OTKA K68792 grant. Address reprint requests and correspondence: Professor Béla Merkely, M.D., Ph.D., D.Sc., Heart Center, Semmelweis University, Városmajor Strasse 68, H-1122 Budapest, Hungary. E-mail address: merkely.bela@kardio.sote.hu. (Received September 14, 2010; accepted January 6, 2011.) reoperation was necessary in only two patients $(0.6 \%)$. Phrenic nerve stimulation was observed in 18 instances, and repositioning with an ablation catheter was performed in seven cases. Impedance measurements did not suggest lead insulation failure. Three stented leads were extracted without complication after 3-49 months owing to infection, while four leads were extracted easily during heart transplantation after 7-27 months.

CONCLUSION Stent implantation to stabilize CS lead position seems to be an effective and safe procedure in prevention and treatment of CS lead dislocation in selected cases.

KEYWORDS Cardiac resynchronization; LV lead implantation; Coronary sinus; Lead dislocation; Stent implantation

ABBREVIATIONS CRT $=$ cardiac resynchronization therapy; CS = coronary sinus; $\mathbf{I N R}=$ international normalized ratio; $\mathbf{L V}=$ left ventricle; $\mathbf{N Y H A}=$ New York Heart Association; PNS $=$ phrenic nerve stimulation

(Heart Rhythm 2011;8:845-850) ${ }^{\odot} 2011$ Published by Elsevier Inc. on behalf of Heart Rhythm Society. lead position. A more stable lead position may increase implantation success rates and decrease the number of postoperative complications. The procedure of CS lead stenting was described elsewhere in detail. ${ }^{8-11}$ In this study, longterm follow-up results of CS stenting are reported in a larger patient population.

\section{Methods}

CS sinus stenting has been performed since August of 2004 in selected patients after receiving informed consent. The consent form and the protocol were approved by the Hungarian Scientific and Research Ethics Committee of the Medical Research Council. Data were collected in consecutive patients who underwent CS lead stabilization with stenting in the Heart Center of the Semmelweis University, Budapest.

CS side branch stenting was performed in 312 patients with wide QRS (>120 ms). New York Heart Association (NYHA) functional stage was mainly III-IV despite optimal medical therapy, while 12 patients were in NYHA II stage at implantation, but their preceding clinical status made this treatment reasonable (Table 1). At our institute, 784 CRT 


\section{Table 1 Baseline Characteristics}

\begin{tabular}{lc}
\hline Mean age & 65.4 \\
Sex: & 236 \\
$\quad$ Male & 76 \\
Female & \\
NYHA class at implantation: & 12 \\
II & 247 \\
III & 53 \\
IV & $28(23-34)$ \\
Left ventricular ejection fraction (\%, median) & \\
Medication (\% of patients): & 36 \\
Aspirin & 24 \\
Clopidogrel or ticlopidine & 48 \\
Coumarin & 14 \\
Aspirin + coumarin & 7 \\
Clopidogrel + coumarin & 12 \\
Aspirin +clopidogrel & 54 \\
Spironolactone & 71 \\
Other diuretics & 87 \\
Beta-blocker & 84 \\
Angiotensin-converting enzyme inhibitors & \\
$\quad$ or angiotensin receptor blockers & \\
\hline
\end{tabular}

systems were implanted between August 2004 and September 2009, and stenting was applied in $39.7 \%$ of CRT patients. Stent fixation of the CS lead was indicated in cases of postoperative dislocation $(\mathrm{n}=16)$. Furthermore, stenting was applied when intraoperative macroscopic or microscopic dislocation occurred or phrenic nerve stimulation (PNS) was observed in a stable anatomical position and the lead needed to be fixed in a more proximal position $(\mathrm{n}=$ 296) (Figure 1).

CS side branch stenting was executed as described elsewhere. ${ }^{9}$ Briefly, after cannulation of the CS ostium with the

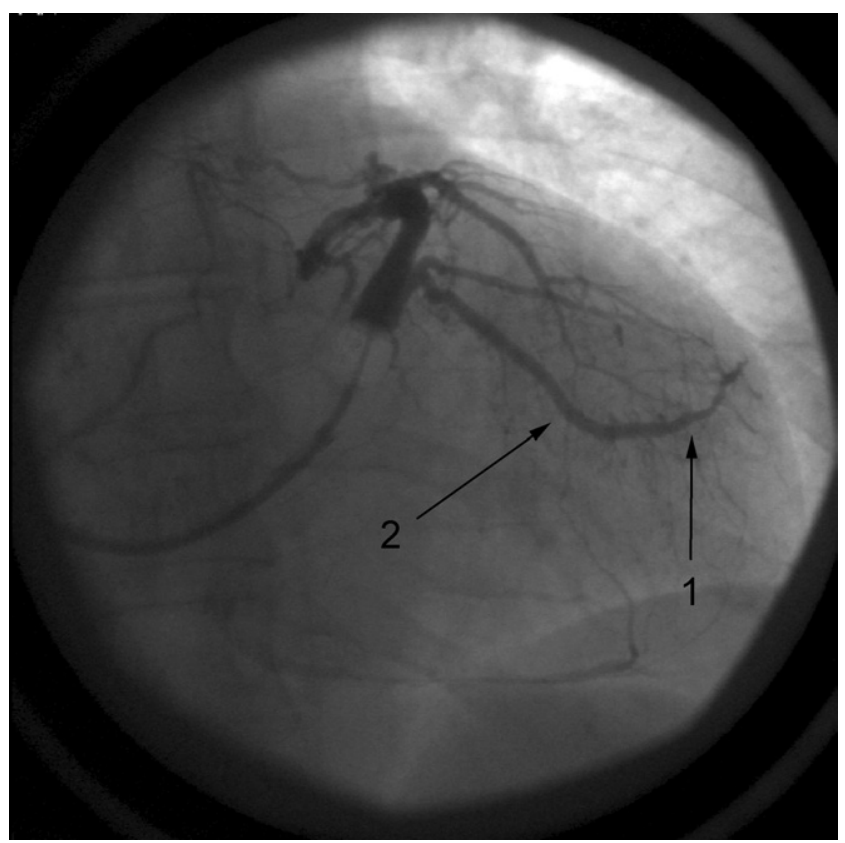

Figure 1 Coronary venogram. Arrow 1: stable, distal wedge position, where PNS was observed. Arrow 2: proximal position with ideal pacing parameters. Intraoperative dislocation was experienced.

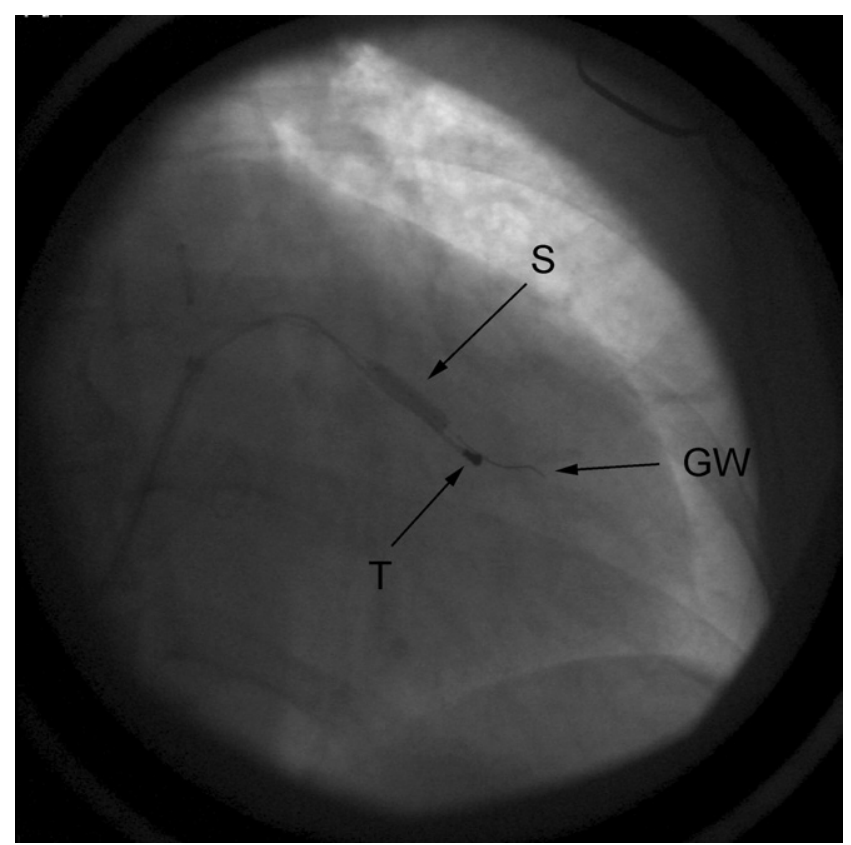

Figure 2 Coronary stent implantation. S: inflation of the stent; GW: guide wire of the stent; T: tip of the lead in the final position.

Scout Pro 8F (Biotronik GmbH\&Co, Berlin, Germany; inner diameter $8 \mathrm{Fr}$ ) or Attain LDS 6216A MB2 (Medtronic Inc., Minneapolis, MN; inner diameter 7 Fr) CS sheaths, CS venography was performed using an occlusion balloon. Generally, over-the-wire left ventricular (LV) unipolar passive fixation electrodes were applied: Attain OTW 4193-78 (Medtronic; $\mathrm{n}=186$ ), Corox OTW 75 UP/Steroid (Biotronik; $\mathrm{n}=118$ ), and Quicksite 1056K-86 (St. Jude, Sylmar, $\mathrm{CA} ; \mathrm{n}=1)$. Seven patients received bipolar passive fixation leads (Corox OTW 75 BP/Steroid, Biotronik). After positioning of the lead, signal amplitude, pacing threshold, and pacing impedance were measured. PNS was assessed in all cases. Repositioning of the LV lead was performed if PNS was apparent during $10 \mathrm{~V}$ at $0.5 \mathrm{~ms}$ pacing. In case the physician performing the implantation decided to use stent implantation, a second guide wire was introduced into the target vein over the same CS sheath. Over this second guide wire, a short (mainly $8-15 \mathrm{~mm}$ ) bare metal coronary stent was positioned into the CS side branch. The distance between the pacing tip (or ring) of the lead and the distal end of the stent was $5-35 \mathrm{~mm}$. The diameter of the stents (2.25-4 mm, mainly 3 or $3.5 \mathrm{~mm}$ ) was chosen according to the diameter of the target CS side branch, which was measured on the CS venogram. The applied bare metal stents were as follows: Trimaxx (Abott Vascular, Redwood City, $\mathrm{CA} ; \mathrm{n}=142$ ), Driver (Medtronic, $\mathrm{n}=57$ ), MicroDriver (Medtronic, $\mathrm{n}=21$ ), S670 (Medtronic, $\mathrm{n}=6$ ), Lekton Motion (Biotronik, $n=35$ ), ProKinetic (Biotronik, $n=29$ ), Liberte (Boston Scientific, Maple Grove, MN, $n=19$ ), and Tsunami Gold (Terumo, Tokyo, Japan, $\mathrm{n}=3$ ). After measuring the control pacing threshold and testing the PNS, the stent was deployed with a pressure of 6-14 atmospheres (Figure 2). The duration of balloon inflation was 4-6 seconds. 
A biventricular pacemaker was implanted in 207 patients, while a biventricular defibrillator was indicated in 105 cases. The decision to use a CRT-P or a CRT-D implantation was at the discretion of the operator. In patients with permanent atrial fibrillation $(n=99)$, only right ventricular and CS leads were implanted.

Additional antiplatelet or anticoagulant therapy was not used after CS side branch stenting; only the preprocedural anticoagulant and/or antiplatelet treatment was continued (Table 1). The antithrombotic treatment was chosen according to current guidelines. The mean daily dosages were the following: aspirin $100 \mathrm{mg}$, clopidogrel $75 \mathrm{mg}$, and ticlopidine $2 \times 250 \mathrm{mg}$. The international normalized ratio (INR) target value was $2-3$ for patients with anticoagulation. However, the INR value was not measured at our hospital because the oral anticoagulation therapy was suspended during the period of device implantation. In the long term, the INR was monitored at the referring hospitals.

Perioperative events were evaluated in all patients. After implantation, patients were seen at the outpatient clinic every 6 months. Median follow-up time was 28.4 (15-38, maximum 70) months. Two hundred ninety-three patients completed 6 months; 186 patients 1 year; 153 patients 2 years; 47 patients 3 years; and 13 patients 4 years of followup. LV pacing threshold and pacing impedance values measured after the implantation were compared with the values recorded during 6-, 24-, and 36-month visits. If the patient experienced PNS, the PNS threshold was confirmed using different pulse width values as well. To minimize PNS, changing the pacing amplitude, pulse duration, or pacing configuration was attempted. If these maneuvers failed to terminate intolerable PNS, minimal invasive lead repositioning was performed. ${ }^{12}$ A steerable ablation catheter (Celsius, B curve, 36H-37R Biosense Webster, Diamond Bar, CA) was introduced into the right atrium via the right femoral vein. The ablation catheter was looped around the atrial part of the CS lead and was retracted together with the CS electrode.

If stented CS leads were extracted for any reason, macroscopic analysis was performed in all cases, looking for injuries on the electrode insulation. When it was possible, microscopic measurements were also taken.

\section{Statistical analysis}

Statistical analysis was prepared with Prism for Windows 5.00 (GraphPad Software, San Diego, CA, www.graphpad. com). Since all of the variables had non-Gaussian distributions, we used nonparametric tests. We used the Wilcoxon signed rank test for comparisons between two repeated measures. All statistical analyses were two-tailed and $P<.05$ was considered statistically significant. Values presented in the text are medians (interquartile ranges), unless otherwise stated.

\section{Results}

CS side branch stenting was successfully performed in 312 (98.4\%) out of 317 patients. In five cases, it was not possible to introduce the stent into the side branch owing to tortuosity of the proximal part or the small diameter of the vessel. In four cases, the electrode was implanted in the side branch without stenting, and in one patient we changed to transseptal endocardial implantation because of intraoperative dislocation. No implantation-related death occurred in our patient group. Mechanical injury caused by CS stenting was not experienced. CS dissection with pericardial tamponade was detected in one patient during implantation. Since contrast dye extravasation was seen in the pericardial space on the first CS venogram before stent deployment, the effusion was presumably caused by CS dissection during positioning of the CS guide. After percutaneous aspiration from a subxyphoideal puncture, the pericardial effusion did not recur.

Early lead dislocation with loss of LV capture was detected in two patients $(0.6 \%)$. Potential causes of the dislocation might be the proximal lead position in a lateral side branch, underestimation of the diameter of the stent, or the localization of the stent in a curvature of the vessel. Reoperation was performed; in one patient, LV pacing was carried out via the anterolateral branch. PNS was detected in seven patients $(2.2 \%)$ during the in-hospital period. Changing the pacing parameters definitively or transiently solved the problem. During follow-up, 54 patients died an average 13.4 months after implantation $(\mathrm{n}=11$ during the first $1-3$ months, $\mathrm{n}=17$ during the 3 month to 1 year period, $\mathrm{n}=16$ during the $1-2$ year period, $\mathrm{n}=10$ after 2 years).

Compared with the values measured after the implantation, the LV pacing threshold did not change significantly after 6 months $(1.0[0.6-1.6]$ vs. $0.8[0.6-1.3] \mathrm{V} ; P=.052$; Figure $3 \mathrm{~A})$ or after 24 months of follow-up $(0.8$ [0.6-1.6] vs. $0.8[0.6-1.5] \mathrm{V}, P=.419$; Figure $3 \mathrm{C}$ ). A clinically remarkable rise in pacing threshold was observed in two cases $(0.6 \%)$. In one patient, the threshold increased after 2 years $(2.2$ vs. $5.6 \mathrm{~V})$. In the other case, the lowest threshold value was $5.8 \mathrm{~V}$ at $0.5 \mathrm{~ms}$ during implantation, which increased to $7.2 \mathrm{~V}$ at $0.5 \mathrm{~ms}$. In two other cases, the threshold increased more than $2 \mathrm{~V}$ but did not exceed $4 \mathrm{~V}$ at 0.5 $\mathrm{ms}$. Macroscopic dislocation was not detected on X-ray in these four patients. Although a slight decrease of the LV pacing impedance was found at both 6-month (600 [522720 ] vs. 550 [475-639] $\Omega, P=.0007$; Figure 3B) and 24 -month visits (608 [535-780] vs. 575 [508-656] $\Omega, P=$ .0181 ; Figure 3D), results of impedance measurements did not suggest insulation failure or fracture of the LV electrode in any cases during follow-up. In patients with $3(n=47$, Figure $3 \mathrm{E}$ and $3 \mathrm{~F})$ or 4 years $(\mathrm{n}=13)$ of follow-up, the pacing threshold remained stable (median 0.9 [0.6-1.65] vs. 0.9 [0.6-1.3] $\mathrm{V}$ and 0.9 [0.7-2] vs. $1.25[0.85-2.1] \mathrm{V}$ at 0.5 , respectively), no signs of lead injury were detected (608 [522-810] vs. 563 [511-676] $\Omega$ and 600 [529-688] vs. 636 [553-709] $\Omega$ ).

PNS was observed in 18 patients (5.7\%). In 11 cases, reprogramming of the pacing parameters was successful to terminate PNS. In seven cases $(2.2 \%)$, repositioning of the 
A. 6 months, LV pacing threshold

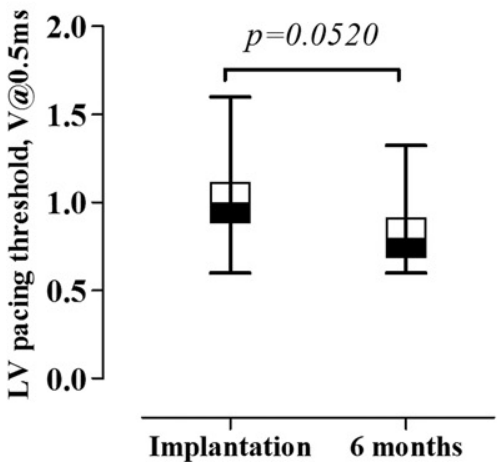

C. 24 months, LV pacing threshold

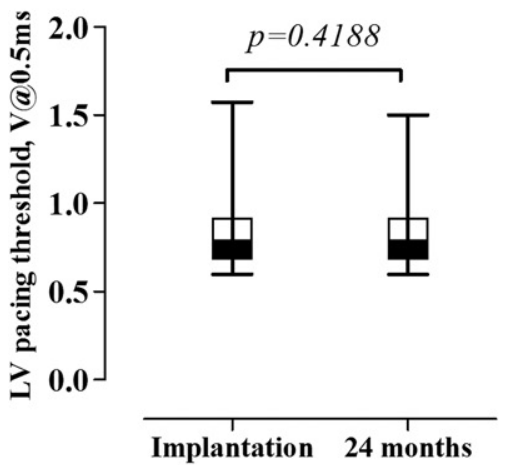

E. 36 months, LV pacing threshold

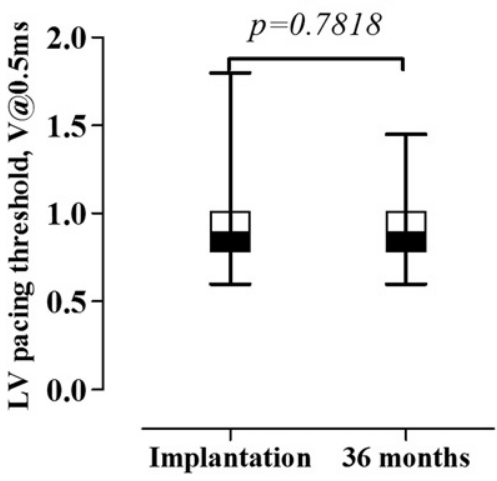

\section{B. 6 months, LV pacing impedance}

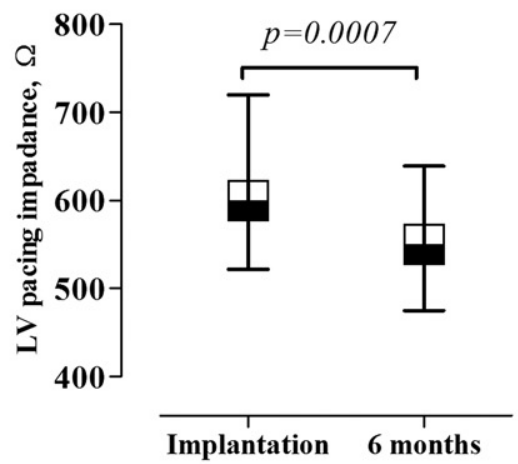

D. 24 months, $L V$ pacing impedance

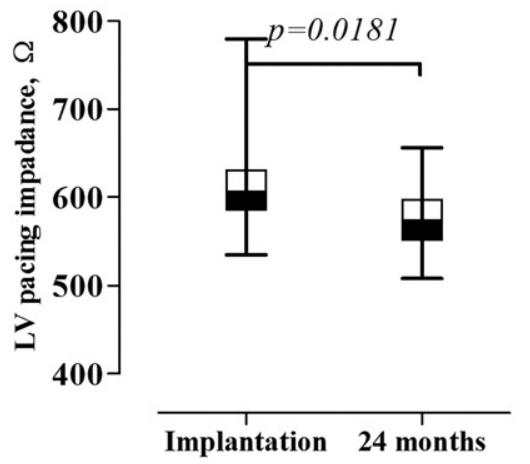

F. 36 months, $L V$ pacing impedance

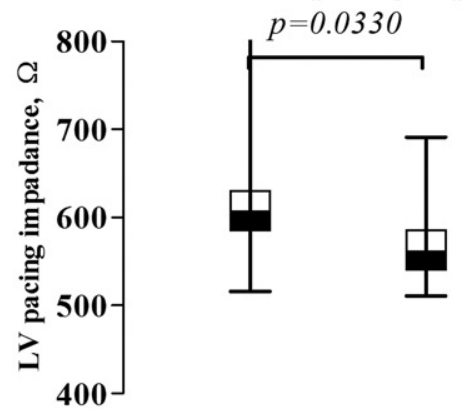

Implantation 36 months
Figure 3 Changes in LV pacing and impedance values at 6,24 , and 36 months after implantation. LV pacing threshold did not change at 6, 24, and 36 months of follow-up, while a slight decrease in the LV pacing impedance values was found without any signs of injury of the lead insulation. ( $n=293$ at 6 months, $\mathrm{n}=153$ at 24 months, $\mathrm{n}=47$ at 36 months). Medians and interquartile ranges are presented. $P$-values were calculated with the Wilcoxon signed rank test. lead was necessary and the stented LV leads were retracted with an ablation catheter introduced via the femoral vein in these patients 1-28 months after implantation. In five cases, adequate pacing threshold was reached, while in one patient the threshold increased to $5.0 \mathrm{~V}$ at $1 \mathrm{~ms}$. In another patient, the CS lead dislocated into the right atrium; it was explanted and a new lead was implanted. PNS was not detected after repositioning.

Explantation of the stented LV lead was needed in three patients because of pocket infection $(\mathrm{n}=2)$ and endocarditis $(\mathrm{n}=1)$ after 3,49 , and 18 months, respectively. Leads were extracted without any complication while the stent remained in the CS side branch. Neither signs of insulation failure nor other macroscopic damage were seen on the extracted electrodes. Four patients underwent heart trans- plantation (7-27 months after implantation). During the operation, leads were cut in the superior vena cava, and after explantation of the heart (Figure 4) the surgeon was able to extract easily the stented leads from the CS side branch. Between the stent and the lead, a macroscopically identifiable layer of tissue was observed (Figure 5). Macroscopic injuries could not be seen. Microscopic evaluation was performed on five CS electrodes (Figure 6). The examination of the stented area revealed surface damage as a result of the continuous friction between the stent and the electrode, with a depth of 3-7 $\mu \mathrm{m}$. This depth involved $1.7 \%-4 \%$ of the total insulation wall thickness. In one lead, a deeper injury (27\% of the insulation thickness) was also found, but the sharp edges and long, narrow shape of the 


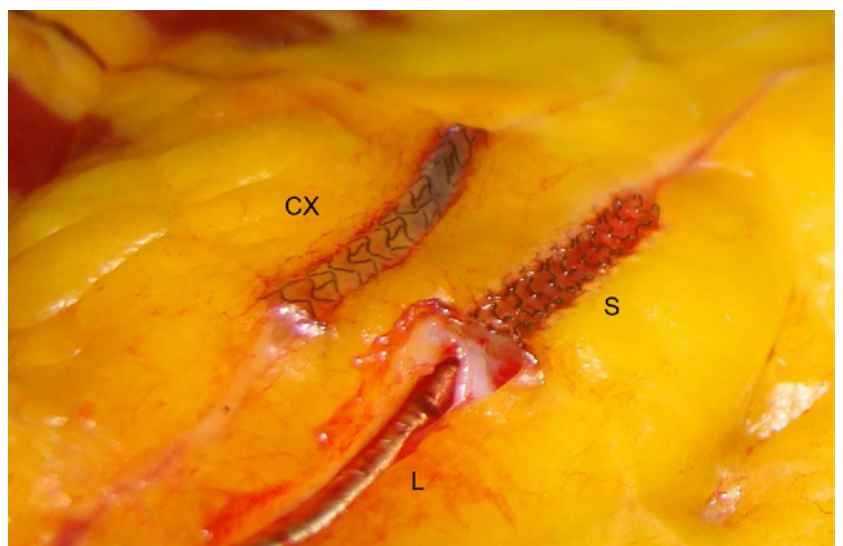

Figure 4 Epicardial view of the explanted heart: L: unipolar electrode in the lateral side branch of the CS; CX: stent in the circumflex artery, which was implantated when primary PCI was performed in nSTEMI during follow-up.

deepest injury suggest that it was produced during the extraction of the lead.

\section{Discussion}

Stable LV lead position in the area of latest activation is important in CRT. ${ }^{13-15} \mathrm{LV}$ lead implantation into the recommended lateral or posterolateral side branch of the CS is not feasible due to anatomical and/or technical limitations in up to one-third of patients. ${ }^{16}$ An important cause of suboptimal lead positioning, lead dislodgement, or extracardiac stimulation is the unstable electrode position in the target vein. ${ }^{17}$ The original idea was that CS stenting may decrease the dislocation rate and improve the success rate of lead implantation into the recommended side branches by anchoring the LV lead in an anatomically unstable position. ${ }^{9}$ CS stent implantation was first used in cases of postoperative lead dislocation. Since complications have not been observed, stenting also has been performed in cases of intraoperative dislocation, unstable lead position, or when

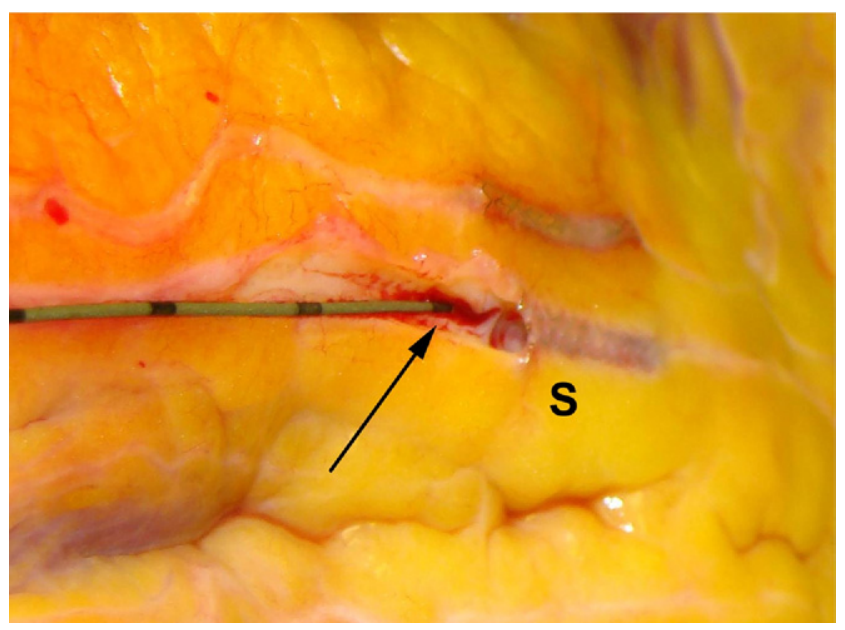

Figure 5 Epicardial view of the explanted heart: S: stent in the lateral side branch of the CS after explantation of the lead during heart transplantation; arrow: place of the explanted CS lead.

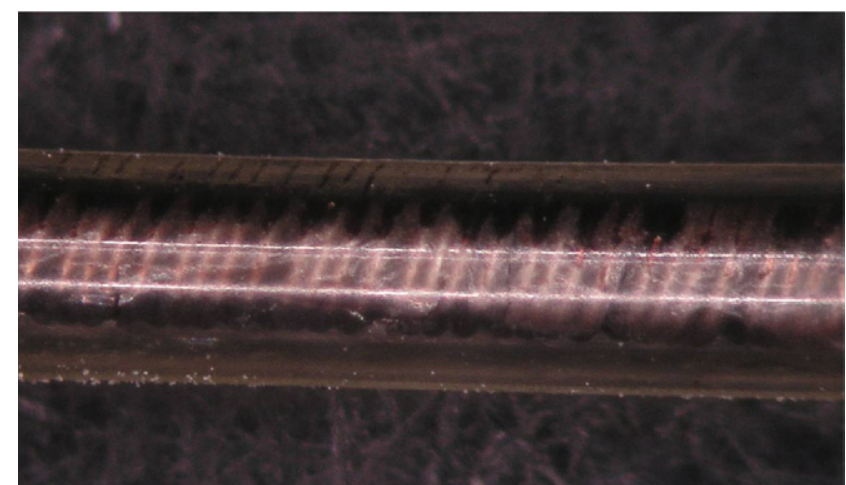

Figure 6 CS lead extracted during heart transplantation. Metal microscopic image about the surface covered by the stent. Injury of the lead insulation was not observed.

PNS was found adjacent to the tip of the CS lead. In these cases, the aim was to prevent potential electrode dislocation.

Stents successfully anchored the CS leads in this patient group. The consistency of pacing thresholds support the stability of the stented leads. Macrodislocation was detected in only two patients. In seven other cases, the cause of lead repositioning was PNS. PNS is found in 13\%-18\% of CRT implantations, and it is one of the main reasons for intraoperative lead repositioning from an anatomically acceptable location. ${ }^{18,19}$ Pacing with high energy during implantation may help to avoid subsequent PNS, but despite high-energy stimulation, intraoperative testing in a supine position cannot rule out later PNS in other body positions, ${ }^{20}$ even if the electrode remains in the same place. In seven cases, a new, minimally invasive method was performed using an ablation catheter for repositioning of the lead via a femoral approach. The pacemaker pocket had to be opened for lead repositioning in only three of our 312 patients $(0.9 \%)$. This ratio is much lower than the rate of reoperation reported in the literature in large multicenter studies. ${ }^{1,21,22}$

Potential complications due to CS stent implantation may be the injury of the target vein and/or mechanical damage of the electrode. Our observations are in line with prior case reports that describe no complications during stent implantation into the venous system of the heart ${ }^{23-25}$ or during stenting to fix the attained electrode position. ${ }^{9-11}$ Mechanical damage of the lead insulation caused by the stent may also be a potential problem, especially over a longer period of time. In our patients, impedance measurements did not suggest insulation failure or fracture of the LV electrode during follow-up. Optical and mechanical microscopic analysis of the explanted CS electrodes revealed only mild surface damage in the area of contact with the stent. The depth of surface injury involved less than $4 \%$ of the total insulation thickness, which suggests that the stent does not jeopardize the integrity of the lead insulation. ${ }^{26}$ On explanted hearts, a fibrotic sheath was observed around the stent enveloping the electrode, which may decrease direct friction between the stent and the lead.

There may be significant concern regarding extraction of a CS lead fixed with a stent should it become necessary. 
Although one might believe that it is only possible by cardiac surgery techniques, ${ }^{27}$ stented CS leads were explanted with simple traction in three patients (in one case after 4 years). In addition, four more electrodes were also easily extracted by traction during heart transplantation. Moreover, we have managed to alter the CS lead position by retraction from a distal position with an ablation catheter in cases of PNS.

Our study is a nonrandomized, uncontrolled single-center clinical experience. In this study, mainly unipolar passive fixation CS leads were implanted. Although our results seem to be favorable, longer term performance of the stented LV leads is unknown. Mechanical damage of lead insulation may occur due to motion between the stent and the implanted lead, but according to our microscopy measurements, the probability of clinically important injury is low. CS stent implantation may limit the ability of lead removal; however, data regarding safe extraction of other actively fixed CS leads (like StarFix Attain 4195, Medtronic) ${ }^{28-30}$ or screw-in pacemaker electrodes in the $\mathrm{CS}^{31}$ are inconsistent or missing. Notably, we have extracted stented LV leads without heart surgery in all of our patients when necessary $(n=3 ; 1 \%)$. Moreover, minimally invasive lead repositioning with an ablation catheter was also successful $(\mathrm{n}=7 ; 2.2 \%)$. Although our implantation success rate was very high, and the dislocation rate of the stented CS leads was low, a direct comparative multicenter study would be beneficial to further clarify the advantages of CS lead stenting.

\section{Conclusion}

CS side branch stenting to stabilize CS lead position seems to be an effective and safe procedure. Using this technique, the electrode can be fixed in an anatomically unstable but electrically appropriate position such that the frequency of lead dislocation, PNS, and reoperations may be lowered. In our practice, stented CS leads were transvenously explantable in all patients when necessary. We endorse CS stent implantation in cases of postoperative or intraoperative lead dislocation or if the electrode position is not stable enough and an alternative side branch is not available at the chosen location.

\section{References}

1. Bristow MR, Saxon LA, Boehmer J, et al. Cardiac-resynchronization therapy with or without an implantable defibrillator in advanced chronic heart failure. N Engl J Med 2004;350:2140-2150.

2. Gras D, Bocker D, Lunati M, et al. Implantation of cardiac resynchronization therapy systems in the CARE-HF trial: procedural success rate and safety. Europace 2007;9:516-522.

3. Leon AR, Abraham WT, Curtis AB, et al. Safety of transvenous cardiac resynchronization system implantation in patients with chronic heart failure: combined results of over 2,000 patients from a multicenter study program. J Am Coll Cardiol 2005;46:2348-2356.

4. Ailawadi G, Lapar DJ, Swenson BR, et al. Surgically placed left ventricular leads provide similar outcomes to percutaneous leads in patients with failed coronary sinus lead placement. Heart Rhythm 2010;7:619-625.

5. Moss AJ, Hall WJ, Cannom DS, et al. Cardiac-resynchronization therapy for the prevention of heart-failure events. N Engl J Med 2009;361:1329-1338.

6. Borleffs CJ, van Bommel RJ, Molhoek SG, de Leeuw JG, Schalij MJ, van Erven
L. Requirement for coronary sinus lead interventions and effectiveness of endovascular replacement during long-term follow-up after implantation of a resynchronization device. Europace 2009;11:607-611.

7. van Gelder BM, Scheffer MG, Meijer A, Bracke FA. Transseptal endocardial left ventricular pacing: an alternative technique for coronary sinus lead placement in cardiac resynchronization therapy. Heart Rhythm 2007;4:454-460.

8. Gellér L, Szilagyi S, Róka A, Merkely B. Coronary sinus sidebranch stenting as a tool for coronary sinus lead dislocation in patients with heart failure and left bundle branch block. Eur Heart J 2005;26(Suppl 1):52/P486.

9. Szilagyi S, Merkely B, Roka A, et al. Stabilization of the coronary sinus electrode position with coronary stent implantation to prevent and treat dislocation. J Cardiovasc Electrophysiol 2007;18:303-307.

10. Kowalski O, Lenarczyk R, Prokopczuk J, et al. Effect of percutaneous interventions within the coronary sinus on the success rate of the implantations of resynchronization pacemakers. Pacing Clin Electrophysiol 2006; 29:1075-1080

11. Cesario DA, Shenoda M, Brar R, Shivkumar K. Left ventricular lead stabilization utilizing a coronary stent. Pacing Clin Electrophysiol 2006;29:427-428.

12. Szilagyi S, Merkely B, Zima E, et al. Minimal invasive coronary sinus lead reposition technique for the treatment of phrenic nerve stimulation. Europace 2008;10:1157-1160.

13. Morgan JM, Delgado V. Lead positioning for cardiac resynchronization therapy: techniques and priorities. Europace 2009;11(Suppl 5):v22-28

14. Merchant FM, Heist EK, McCarty D, et al. Impact of segmental left ventricle lead position on cardiac resynchronization therapy outcomes. Heart Rhythm 2010;7:639-644.

15. Singh JP, Fan D, Heist EK, et al. Left ventricular lead electrical delay predicts response to cardiac resynchronization therapy. Heart Rhythm 2006;3:1285-1292.

16. Yu CM, Wing-Hong Fung J, Zhang Q, Sanderson JE. Understanding nonresponders of cardiac resynchronization therapy-current and future perspectives. J Cardiovasc Electrophysiol 2005;16:1117-1124.

17. Lau EW. Achieving permanent left ventricular pacing-options and choice. Pacing Clin Electrophysiol 2009;32:1466-1477.

18. Gurevitz O, Nof E, Carasso S, et al. Programmable multiple pacing configurations help to overcome high left ventricular pacing thresholds and avoid phrenic nerve stimulation. Pacing Clin Electrophysiol 2005;28:1255-1259.

19. Schwierz T, Winter S, Pürerfellner H, Tomaselli F, Nesser H, Függer R. Nervus Phrenicus Stimulation bei biventrikulären Schrittmachern. Chirurg 2007;78: 1037-1040.

20. Azizi M, Castel MA, Behrens S, Rodiger W, Nagele H. Experience with coronary sinus lead implantations for cardiac resynchronization therapy in 244 patients. Herzschrittmacherther Elektrophysiol 2006;17:13-18.

21. McAlister FA, Ezekowitz JA, Wiebe N, et al. Systematic review: cardiac resynchronization in patients with symptomatic heart failure. Ann Intern Med 2004;141:381-390.

22. Cleland JG, Daubert JC, Erdmann E, et al. The effect of cardiac resynchronization on morbidity and mortality in heart failure. N Engl J Med 2005;352: $1539-1549$.

23. van Gelder BM, Meijer A, Basting P, Hendrix G, Bracke FA. Successful implantation of a coronary sinus lead after stenting of a coronary vein stenosis. Pacing Clin Electrophysiol 2003;26:1904-1906.

24. Scott PA, Chantrarat T, Roberts PR, Curzen NP. Percutaneous coronary venous stenting to facilitate placement of a left ventricular lead. Int J Cardiol 2009;133: e76-78

25. Gutleben KJ, Nolker G, Marschang H, et al. Rescue-stenting of an occluded lateral coronary sinus branch for recanalization after dissection during cardiac resynchronization device implantation. Europace 2008;10:1442-1444.

26. Balázs T, Merkely B, Bognár E, et al. Methods for examination an explanted left ventricular pacemaker lead stabilized with a coronary stent. Pacing Clin Electrophysiol 2010 Nov 11. doi: 10.1111/j.1540-8159.2010.02955.x.

27. Hansky B. Stenting of coronary veins: a critical comment. Europace 2008; 10:1363.

28. Bongiorni MG, Di Cori A, Zucchelli G, et al. A modified transvenous single mechanical dilatation technique to remove a chronically implanted active-fixation coronary sinus pacing lead. Pacing Clin Electrophysiol 2010 May 11. doi: 10.1111/j.1540-8159.2010.02784.x.

29. Crossley GH, Exner D, Mead RH, et al. Chronic performance of an active fixation coronary sinus lead. Heart Rhythm 2010;7:472-478.

30. Nagele H, Azizi M, Hashagen S, Castel MA, Behrens S. First experience with a new active fixation coronary sinus lead. Europace 2007;9:437-441

31. Hansky B, Vogt J, Gueldner H, et al. Implantation of active fixation leads in coronary veins for left ventricular stimulation: report of five cases. Pacing Clin Electrophysiol 2007;30:44-49. 